\title{
La régulation de l'éducation en France et dans les pays anglo-saxons: une comparaison
}

\section{Denis Meuret}

L'autonomie des établissements s'est accrue dans les vingt dernières années dans la plupart des systèmes éducatifs. Ces derniers ont donc dî mettre en place des politiques de régulation pour orienter l'activité des agents dans les directions souhaitées par la nation. Alors que l'Angleterre et les Etats-Unis ont mis en place une régulation fondée sur les résultats (la distribution d'incitations, positives ou négatives, en fonction des performances des établissements scolaires ou des enseignants), la France joue plutôt d'une régulation par les processus (l'incitation à mettre en place des "formes pédagogiques» jugées pertinentes par les échelons centraux). Ces politiques sont ici décrites, puis confrontées du point de vue de leur cohérence et des tensions qu'elles suscitent, dans l'esprit de savoir si le modèle français est un modèle viable, alternatif au modèle anglo-saxon. La réponse est plutôt négative, en même temps quill apparaî̀ que les pays anglo-saxons eux-mêmes mêlent de plus en plus les deux modes de régulation.

L'organisation traditionnelle des systèmes éducatifs des pays anglo-saxons repose sur une base locale, accorde une grande influence aux usagers, laisse une grande marge d'autonomie aux enseignants dans la construction du curriculum. Elle differe profondément de l'organisation traditionnelle du système français: centralisée, bureaucratique, dotée d'un programme national contraignant fortement les curricula.

Depuis une vingtaine d'années, cependant, ces organisations se sont en vérité rapprochées, illustrant la convergence des systèmes éducatifs modernes. L'emprise nationale sur les curriculum s'est accrue dans les pays anglo-saxons, à travers des épreuves communes proposées par les Etats américains ou à travers le «national curriculum» anglais (Davies \& Guppy, 1997). De l'autre côté, l'autonomie des établissements français s'est accrue, des outils de gestion ont été empruntés à la culture anglo-saxonne (les projets d'établissements trouvent pour partie leur origine dans les School development plans britanniques), des politiques se sont implantées dont l'objet est d'adapter le fonctionnement des établissements à leur contexte (celle des Zones d'Education Prioritaires en est l'exemple 
le plus connu). Toutes ces organisations se sont rapprochées d'un modèle dans lequel le Centre définit les contenus et les examens tandis que s'accroît l'autonomie pédagogique ou organisationnelle des établissements scolaires.

C'est bien de convergence qu'il s'agît, puisqu'il ne s'agit pas du transfert autoritaire d'un modèle dans un pays, comme ceux qui ont pu accompagner les colonisations. Cependant, il y a bien emprunts, mais croisés, et ces emprunts s'accompagnent eux aussi d'une adaptation, une «indigénéisation», des formes importées, qui peut être plus ou moins réussie (Schriewer, 1997). Ainsi, le premier curriculum proposé en Angleterre suscita une opposition forte et ne fut mis en œuvre que sous une forme atténuée et concertée (Stearns, 1996). On se propose ici d'étudier un aspect particulier de cette convergence: le type de régulation qui accompagne, dans ces deux types de pays, la nouvelle organisation, en appelant régulation la façon dont une autorité reconnue s'y prend pour orienter les actions et les comportements des acteurs sur lesquels elle a autorité (Meuret \& Duru-Bellat, 2002).

On poursuit ainsi une réflexion sur la régulation des systèmes éducatifs commencée par une revue de la littérature, réalisée avec des collègues de l'Institut de recherche sur l'Economie de l'Education de Dijon (IREDU) pour le Comité National (français) de la Recherche en Education (Meuret, Broccholochi \& DuruBellat, 2001; Duru-Bellat \& Meuret, 2001). Après avoir confronté les effets des deux modèles de régulation sur l'efficacité et l'équité de l'enseignement (Meuret \& Duru-Bellat, 2002), puis leurs fondements théoriques (Meuret \& Duru-Bellat, 2003), on voudrait ici les comparer sous l'angle de leur cohérence interne, des contradictions ou tensions dont ils sont porteurs.

On présentera donc les formes que prend l'autonomie des établissements d'une part aux Etats-Unis et en Angleterre ${ }^{1}$, d'autre part en France, puis les deux formes qu'y prend la régulation, avant de se poser la question de leurs cohérences respectives. En arrière plan de cette comparaison se trouve la question de savoir si l'on peut considérer que la France a réussi à proposer en la matière une alternative crédible à la régulation à l'anglo-saxonne.

\section{Formes de l'autonomie...}

\section{... dans les pays anglo-saxons}

En Angleterre, les établissements jouissent, en échange d'une surveillance étroite de leurs performances, d'une autonomie de moyens considérable en matière de pédagogie ou de gestion du personnel: ils recrutent leurs enseignants eux mêmes, ce que ne font aux Etats-Unis que les Charter schools. Cependant, leur autonomie pédagogique est limitée, pour les disciplines principales, par le curriculum national et, qui plus est, mise entre parenthèses deux heures par jour dans le cadre des National Literacy \& Numeracy Strategies: Tous les enseignants y suivent le même canevas national très précis, mis au point très soigneusement, ce qui évoque 
l'école française très centralisée de la Troisième République davantage que la tradition anglaise d'autonomie professorale ou que la concurrence libre des établissements scolaires sur le marché éducatif.

Aux Etats Unis, l'autonomie des établissements peut prendre, soit la forme du «School Based Management», auquel cas les écoles restent dans le moule commun tout en bénéficiant d'un peu plus d'autonomie, soit la forme d'écoles disposant de l'autonomie attachée à un statut particulier (Magnet schools, Charter schools, Whole school designs).

En 1989, dans les zones touchées par des programmes de School Based Management, les établissements disposaient en fait d'une autonomie bien moindre que celle dont disposent aujourd'hui la totalité des établissements anglais, suédois ou néerlandais. Par exemple, 37\% d'entre eux seulement recrutaient eux mêmes leur personnel (Heller et al., 1989, cité par Murphy \& Beck, 1995 p. 47).

Les Magnet schools sont apparues en 1975. Elles offrent des programmes attrayants dans les quartiers populaires, pour lutter contre la ségrégation. De fait, les Magnet schools sont fréquentées par des élèves volontaires et ont donc représenté une des premières formes de "choix de l'école» aux États Unis. Elles scolarisent aujourd'hui 1,2 millions d'élèves (Du Bois, Eaton, Garet \& Miller, 2000).

Les Charter schools sont des établissements financés sur la même base que les établissements publics standards. Ils passent un contrat avec le district, au terme duquel ils sont libres de leurs décisions dans une série de domaines, en échange de quoi ils acceptent certaines règles (non discrimination, ou encore l'obligation de tirer au sort parmi leurs candidats, par exemple) et d'être évalués périodiquement, cette évaluation étant nécessaire au renouvellement de leur contrat. Les Charter schools sont beaucoup plus autonomes que les établissements des programmes de SBM: 88\% recrutent leurs enseignants, 83\% établissent librement leur programmes d'étude, $75 \%$ organisent librement l'année scolaire (Nelson, 2000). Apparues en 1992, elles scolarisaient, à l'automne 2000, 500'000 élèves et en 2004, 700000 élèves, soit à peu près 1,5\% des élèves américains.

On peut considérer les «Whole school designs» comme une autre forme de l'autonomie. Avec l'accord du district, certaines écoles peuvent implanter des «formes scolaires globales», élaborées le plus souvent par des fondations ou des centres de recherche en éducation (Success for all, par exemple, cf. Slavin et al., 1996). Ces "designs» ont profité de la réorientation de la politique d'éducation compensatoire américaine, en 1994: au lieu de fournir un enseignement spécial aux élèves ciblés par cette politique, on s'efforce d'améliorer l'efficacité globale de leurs établissements, qui peuvent donc utiliser les subventions fédérales à la mise en œuvre de ces «designs». En outre, une décision ultérieure du Congrès (ObeyPorter amendment) a permis à n'importe quel établissement dont les performances sont faibles de recevoir des subventions fédérales pour implanter certains d'entre eux (Herman,1999). La décision d'y recourir est une décision autonome de l'école - la plupart des fournisseurs n'acceptent d'implanter leur programme dans une école que si $80 \%$ de ses enseignants ont voté pour à bulletins secrets - 
mais ensuite celui ci définit assez précisément la façon dont l'école doit fonctionner. Selon certaines études (Springfield, 2000) ces programmes ont de meilleurs effets que les programmes développés au niveau des écoles elles mêmes. Souvent, en effet, ces derniers en restent à la phase de planification et ne s'implantent pas vraiment, ou bien sont le fait de quelques enseignants et ne suivent pas l'élève tout au long de son trajet dans l'école. D'autres évaluations sont moins positives: Dans un district pauvre du Texas, les écoles sous «designs» ne font pas mieux que les autres si l'on tient sous contrôle les caractéristiques des élèves (Berends, Chub, Schuyler, Stockly \& Briggs, 2002). En 1999, huit mille établissements américains du premier ou du second degré avaient implanté l'un ou l'autre de ces designs. Aujourd'hui, 29 d'entre eux sont encouragés par le Ministère dans le cadre des "Compréhensives school reforms». Plusieurs ont fait l'objet de plusieurs évaluations rigoureuses et positives dans des contextes sociaux et académiques différents, prenant en défaut la thèse selon laquelle tout changement doit être spécifique à son contexte, et, plus généralement, la thèse qui fait des "capacités d'adaptation» des écoles à leur public le critère de leur réussite (Borman, Hewes, Overman \& Brown, 2003).

\section{... en France}

On ne trouve pratiquement pas, en France, d'établissements aussi autonomes que les Charter schools ou les écoles privées américaines, puisque les établissements catholiques ont renoncé à l'essentiel de leur autonomie en échange du paiement de leur personnel par l'Etat (Loi Debré). Les seuls qui existent sont quelques rares écoles privées dîtes "hors contrat d'association avec l'Etat» et quelques établissements publics dits, assez hypocritement d'ailleurs, «expérimentaux», un adjectif qui dit bien que la différence n'est tolérée que si on peut faire semblant de croire qu'elle contribue à la production d'une future norme nationale.

Les établissements n'ont aucune prérogative pour la gestion de leur personnel. En revanche, ils jouissent de certaines marges de manœuvre en matière pédagogique, mais, même dans ce domaine, «la marge d'autonomie est celle que les autorités veulent bien lui laisser». Leur Conseil d'Administration est présidé par le chef d'établissement qui est le représentant de l'Etat et il délibère dans le cadre des objectifs fixés par les autorités compétentes de l'Etat (IGEN-EVS, 2001). L'autonomie a été présentée en France comme le corollaire d'une politique qui visait à rattraper le retard du pays en matière de scolarisation du second degré, au moyen d'une argumentation qui en appelait au professionnalisme et à la bonne volonté des enseignants: Pour que puisse réussir le plus grand nombre des élèves, il fallait donner aux enseignants les moyens d'adapter leur enseignement aux besoins de ces derniers. Cela supposait cependant que les enseignants cessent de raisonner uniquement dans le cadre de leur discipline. Il s'agissait, ensemble, de faire réussir les élèves. 
L'autonomie des établissements a donc un double aspect: d'une part, le corset national a été desserré, d'autre part les enseignants ont été invités à se servir collectivement des marges de manœuvre ainsi offertes.

Le desserrement du corset national s'est effectué de façon très progressive, comme s'était faite de façon très progressive ${ }^{2}$ l'autre grande réforme du demisiècle, celle qui a unifié l'enseignement secondaire au sein des collèges et des lycées. Il a commencé au milieu des années 70 et se poursuit aujourd'hui. Il a pris essentiellement trois formes:

- Au lieu que tout soit prescrit par les échelons supérieurs de l'administration, l'établissement se voit affecté une enveloppe de moyens, fixé un cadre, au sein duquel il peut décider lui-même: certaines heures d'enseignement sont «non affectées» et c'est à l'établissement de décider dans quelle discipline elles seront enseignées, si toutefois le rectorat dispose des ressources correspondants. Dans la limite des moyens qui lui sont attribués, l'établissement peut décider de la structure des groupes qu'il met en place, par exemple préférer implanter les classes les plus petites possibles à implanter des classes plus grandes qui de ce fait libèrent des moyens et permettent de multiplier des enseignements optionnels ou facultatifs.

- Sous diverses appellations («modules», «aide individualisée», «travaux personnels encadrés» dans les lycées; dans les collèges, «études dirigées», "travaux croisés», "parcours diversifiés», «itinéraires de découverte»), est apparu un temps d'apprentissage dont les enseignants doivent décider ensemble, au niveau de l'établissement, le contenu. Ce temps est resté modeste (de l'ordre de deux à trois heures par semaine), mais il tend à s'accroître (suscitant à chaque fois les protestations de ceux qui y voient le signe d'un renoncement à l'excellence) au détriment du temps d'apprentissage déterminé par les programmes nationaux. En revanche, des procédures sont fixées aux enseignants, qu'ils doivent suivre, pour fixer ces contenus: faire passer aux élèves de 8, 11 et 15 ans, en début d'année,des épreuves identiques sur tout le territoire national, en analyser en commun les résultats, proposer au conseil d'administration de l'établissement des stratégies pour résoudre les problèmes mis en lumière, etc. On peut en rapprocher des heures animées par les enseignants mais consacrés, plutôt qu'à l'enseignement, à la vie démocratique de la classe, ou à des interventions éducatives: sécurité routière, drogue, tabac, etc.

- que d'imposer, le ministère propose aux établissements des «formes pédagogiques», relativement définies, mais que les établissements sont libres d'implanter ou non. Des exemples en sont, les classes relais, i.e. des classes qui accueillent temporairement les élèves très perturbateurs ou violents ou encore les «troisième d'insertion professionnelle», etc. Il s'agît en général de dispositifs pour les élèves en difficulté.

Cela peut être résumé en disant que le développement de l'autonomie des établissements a consisté en France à remplacer certaines prescriptions détaillées par 
un cadre d'action, certaines règles substantives par des règles procédurales et certaines obligations par des possibilités.

L'incitation à travailler ensemble a pris essentiellement la forme du "projet d'établissement». Il a été rendu obligatoire pour tous les établissements scolaires par la loi du 14 juillet 1989. Le "projet», conformément à la logique de l'adaptation, doit «traduire, pour mieux les assurer, les objectifs nationaux dans leur contexte particulier", il "définit les modalités particulières de mise en oeuvre des objectifs et des programmes nationaux» (Loi du 14.7.1989). Celui-ci définit donc l'autonomie des établissements français comme une autonomie de moyens Il importe cependant de noter que les enseignants ne sont contraints à rien par les textes qui définissent le projet: on escompte qu'ils découvriront les mérites et l'intérêt du travail en commun. Par ailleurs, le cœur de leur activité - l'enseignement des disciplines aux élèves qui ne présentent pas de difficultés particulières - reste hors champ.

\section{La tutelle des établissements autonomes...}

La régulation des établissements peut se fonder sur les procédures qui organisent les relations des établissements scolaires avec leur tutelle administrative ou leurs relations avec leurs usagers. Il sera seulement question ici des premières. Dans les pays anglo-saxons, les procédures se fondent plutôt sur les résultats obtenus par les établissements; en France, plutôt sur les processus qu'ils mettent en œuvre.

\section{... dans les pays anglo-saxons,}

L'évaluation par les résultats repose sur des procédures par lesquelles la tutelle a connaissance des résultats obtenus (Accountability) et en déduit un ensemble d'incitations (incentives) ou d'aides en direction des établissements.

Un des éléments centraux de la régulation anglaise - l'inspection des établissements - emprunte à la fois à la régulation par les processus et par les résultats, puisque le jugement prononcé sur l'école se fonde sur un examen de ces deux dimensions. Depuis 1993, les établissements ont été inspectés, d'abord tous les quatre ans, maintenant tous les six ans, par l'Office for Standards in Education (OFSTED): les inspecteurs rencontrent les parents hors la présence du personnel de l'école, puis passent une semaine dans l'école, où ils prennent connaissance des résultats aux tests, inspectent les cours et l'ensemble de la vie de l'établissement, selon des critères publics (OFSTED, 2000). Les établissements jugent pertinente et utile la liste des critères, mais se plaignent du stress généré par l'inspection et du fait qu'elle ne tient pas assez compte des conditions difficiles qu'ils rencontrent (Riley \& Rowles, 1997). Les chercheurs, eux, se sont demandés pourquoi l'Inspection se dispensait des exigences qui sont celles de la recherche, et qui voudraient par exemple qu'on ne juge une école que d'après un échantillon représentatif de cours, et ont relevé tout ce qui distingue une Inspection d'une 
approche, sinon scientifique, du moins rigoureuse, d'un établissement scolaire (Fitz-Gibbon \& Stephenson, 1996). Ils ont aussi tout simplement mesuré si l'on observait une amélioration des performances des élèves après le passage des inspecteurs, ce qui n'est pas le cas (Rosenthal, 2004).

L'OFSTED insiste aujourd'hui sur l'intérêt pour les écoles de préparer une inspection par un processus d'auto-évaluation et sur la complémentarité des deux processus, peut être parce que l'on a montré que l'auto-évaluation pouvait produire une investigation plus approfondie et sans doute plus susceptible d'être utilisée pour l'amélioration de l'école (MacBeath, 1999). Cette dernière est susceptible de prendre des formes très diverses, certaines fondées surtout sur la mesure des performances académiques des élèves et l'usage d'indicateurs chiffrés et de questionnaires, d'autres plus attentives à la qualité générale du fonctionnement de l'école, au développement personnel des élèves et professionnel des enseignants, utilisant plus volontiers la confrontation réglée des opinions entre acteurs (Saunders, 1999; Macbeath et al., 2000, Meuret \& Morlaix, 2003).

Par ailleurs, tous les élèves passent à 7,11 et 14 ans des épreuves nationales sur les matières concernées par le programme national, avant de passer le CGSE ( $G e$ neral Certificate of Secondary Education) à 16 ans. Des objectifs nationaux sont fixés quant à la proportion d'une génération qui doit atteindre, à tel âge, le niveau requis. Les établissements reçoivent chaque année un rapport, dit «PANDA (Performance AND Assesment) report», qui compare les scores qu'ils ont obtenus à ceux d'établissements similaires. Celui-ci est inséré dans l'Autumn Package, qui leur donne aussi les références nationales et des indications pour les aider à améliorer leurs performances. Cette situation a généré plusieurs instruments de diagnostic, beaucoup plus sophistiqués que le $P A N D A$, mis au point par des centres de recherche, et fournis aux établissements abonnés, qui doivent évidemment fournir aux centres les données nécessaires. Dans le Lancashire, la LEA a étendu l'usage d'un de ces instruments à tous les établissements de son ressort (Thomas, 2001).

S'agissant des incitations, on sait que l'Angleterre a implanté un système de salaire au mérite pour conserver dans l'enseignement ses meilleurs professeurs. Par ailleurs, les enseignants les plus inefficaces restent rarement en place s'ils ne s'améliorent pas (Wragg, 1999): ils partent à la retraite, sont licenciés, ou, plus rarement, changent d'établissement. Quant aux établissements, ceux que l'OFSTED juge en dessous du minimum admissible sont réinspectés, et, si le premier jugement est confirmé, ils sont mis sous une procédure («Fresh start») par laquelle l'établissement passe sous la tutelle de la LEA, est contraint d'appliquer un plan de redressement, sous la surveillance fréquente d'Inspecteurs et sous la direction d'un nouveau chef d'établissement dans environ la moitié des cas. Fin 1996, sur les 90 établissements passés depuis 1993 par ce dispositif, 12 avaient été fermés, 14 étaient revenus dans le droit commun, et 63 avaient progressé, mais pas assez pour être retirés du dispositif (Stoll \& Myers, 1997). Plus récemment, à ce système d'incitations négatives, s'est ajouté un système d'incitations 
positives qui joue sur une des aspirations les plus fortes des enseignants anglais: les établissements les plus performants bénéficient d'une autonomie pédagogique accrue.

Il n'existe pas d'Inspection aux Etats Unis, sauf pour l'accréditation des écoles. Cependant, l'Etat de Rhode Island met en œuvre une intéressante procédure "d'inspection par les pairs", qui se focalise sur les processus en œuvre dans les classes selon une méthodologie codifiée et dans laquelle l'équipe d'inspecteurs est composée majoritairement d'enseignants en activité dans d'autres établissements et dirigée par l'un d'eux (Wilson, 2001).

Les Etats ont multiplié les "assessments», dont les résultats sont, toujours, renvoyés aux écoles pour qu'elles se comparent avec les autres, et, souvent, publiés, comme le sont, en Angleterre, les résultats du GCSE dans les "Leagues tables». "Quarante huit Etats testent maintenant leurs élèves, dix-neuf exigent que le diplôme de fin d'études secondaires soit donné à partir d'examens de l'Etat, trente six publient des tableaux de bords (report cards) de chaque établissement, dixneuf publient des listes d'établissements peu efficaces, et quatorze récompensent financièrement les établissements qui ont de bonnes performances " (Skrla et al., 2000). Le plus souvent, les performances publiées sont brutes, ce qui suscite l'indignation de beaucoup d'enseignants, puisque l'inégalité des chances de réussite des élèves des différents établissements n'est pas prise en compte. De ce fait, certains états ont prévu de publier des indicateurs "corrigés», dits de "valeur ajoutée», notamment la Floride. La loi fédérale de 2001, "No child left behind» est la généralisation de procédures de ce type. Elle fait obligation aux Etats de mesurer régulièrement les performances de leurs élèves et les incite à installer une série d'actions d'aide, de pressions et de sanctions pour les établissements dont les élèves ne font pas des "adequate yearly progress». Par exemple, à New York, le district finance des associations pour qu'elles offrent des séances de soutien scolaire aux élèves des écoles dont les performances sont insuffisantes. Il est à noter que le système d'indicateurs mesure que les élèves de toutes les catégories ethniques bénéficient également de ces progrès.

Dans quelques districts, des récompenses sont attribuées aux établissements en fonction des résultats obtenus aux tests. Elles peuvent leur être attribuées en fonction des performances moyennes de leurs élèves, des performances de leurs élèves appartenant à des minorités, en fonction aussi de l'évolution de ces performances, ou encore d'indices comme le taux d'absentéisme des élèves ou la proportion d'élèves qui quittent la système scolaire (drop outs). Elles sont redistribuées également à chaque enseignant ou membre du personnel de l'établissement (District de Dallas), ou bien selon une clé qui doit être «décidée démocratiquement» (Kentucky, avant 1998) entre ses agents, ou bien encore attribuées à l'établissement en général pour son fonctionnement (Ladd, 1999; Pedrosko, 2000). Aucun de ces systèmes ne prévoit de distinguer entre les personnels de l'établissement selon leur contribution à l'efficacité d'ensemble. Consultés sur 
leurs souhaits par l'Education Commission of the States en 2003, huit responsables de districts exemplaires ont suggéré, entre autres, un système à l'anglaise, libérant les écoles les plus efficaces de la tutelle du district, ainsi rendu capable de se consacrer davantage aux écoles en difficulté.

Il existe des systèmes d'incitation adressés directement aux enseignants, mais ils sont très rares. Le district de Cincinatti est apparemment le premier à avoir signé un accord à ce sujet avec les syndicats d'enseignants, en septembre 2000, accord qui prévoit la mise en place de ce système pour 2002 (Blair, 2000).

Des dispositifs drastiques d'incitations négatives, parfois ordonnés par des tribunaux, ont été expérimentés. Dans quelques districts, lorsqu'un établissement est jugé par trop inefficace, l'ensemble de son personnel est remplacé, pour, disent les promoteurs de ce dispositif, «briser la spirale de la résignation» (Reconstitution). C'est par exemple le cas dans certains districts du Maryland et dans le district de San Francisco ${ }^{3}$.

\section{... en France}

Les textes administratifs qui organisent la fonction publique française depuis 1945 prévoient que les agents puissent être sanctionnés. Pour les enseignants, contrairement à ce qu'on croit souvent, il existe en France un système de «salaire au mérite»: lors de sa visite, plus ou moins fréquente selon les endroits et les disciplines, l'inspecteur met à chaque enseignant une note qui détermine la vitesse avec laquelle il franchit les différents échelons de sa carrière. En fin de carrière, entre ceux qui ont franchi les échelons le plus vite et le plus lentement possible, l'écart des sommes reçues comme salaire est important.

Mais ces récompenses sont attribuées aux enseignants qui mettent en pratique des processus que les inspecteurs estiment souhaitables, non pas à ceux qui obtiennent les meilleurs résultats avec leurs élèves. Cela n'empêche donc pas que la culture du résultat soit profondément contraire à la culture de l'administration, particulièrement dans le système éducatif, où, plus qu'ailleurs peut être, on peut faire état de la difficulté à mesurer les résultats de façon sûre et juste.

Les chefs d'établissement (ainsi que les parents et les élèves) se plaignent fréquemment qu'il est très difficile d'engager quelque action que ce soit en direction des enseignants vraiment inefficaces. Par ailleurs, aucune procédure particulière n'est prévue en cas de faible performance d'un établissement. Lorsque des inspections d'établissements ont lieu, on précise bien que les conclusions à en tirer sont à la discrétion de l'établissement lui-même. Ces conclusions ne sont jamais publiques. Lorsque l'administration tente de passer avec les établissements des «contrats de réussite», il se produit ce que Demailly (2003) décrit ainsi: "On est passé en un an d'une philosophie de l'évaluation définie comme un contrôle des contrats de réussite à l'idée d'une aide, d'un accompagnement et d'une écoute des bilans d'étape». Des indicateurs (IPES, Indicateurs pour le Pilotage des Etablissements Secondaires) sont envoyés par le ministère aux établisse- 
ments. Ils leur permettent d'apprécier, relativement aux autres établissements de même type, les caractéristiques sociales et scolaires de leurs élèves, celles de leurs enseignants, l'ampleur des ressources dont ils disposent, et de l'efficacité avec laquelle ils préparent les élèves aux examens (indicateurs dits de «valeur ajoutée»). Mais ils sont conçus comme des outils à l' usage propre des établissements et non comme l'instrument de leur contrôle par les rectorats. Ils sont, de (ce?) fait, rarement utilisés par les établissements.

De même, les évaluations des élèves ont lieu en début, et non en fin, d'année scolaire, au début, et non en fin, de la scolarité dans les collèges et dans les lycées. Ainsi sont-elles un outil pédagogique pour l'enseignant qui accueille l'élève sans pouvoir servir à évaluer le travail de celui qui l'a enseigné ou la qualité de l'établissement qu'il quitte.

Toutefois, deux dispositifs locaux ressemblent davantage à l'accountability anglo-saxonne. D'une part, quelques inspecteurs d'académie rencontrent annuellement chaque principal ${ }^{4}$ de leur département, examinent avec lui les indicateurs qui mesurent le succès de leurs élèves (orientation, redoublement, absentéisme, violence, etc.) et les politiques qu'il entend suivre pour, si c'est nécessaire, faire face à ses difficultés Par ailleurs, dans une académie ${ }^{5}$, les relations entre les établissements du second degré et les rectorats prennent la forme de contrats pluriannuels, élaborés après un diagnostic de l'établissement, dans lesquels le rectorat s'engage à apporter des ressources (formation, personnels) et l'établissement à implanter des politiques poursuivant certains objectifs et à mesurer leur réussite. La circulaire ministérielle qui organise la rentrée 2003 préconise l'extension de ce système qui "contribue à une responsabilisation des acteurs», mais il est trop tôt pour savoir si cette injonction a été suivie d'effets. Les précédents, par exemple un dispositif d'audit imaginé par une Commission Nationale en 2000 et jamais mis en œuvre, laissent dubitatif à cet égard.

De quelle régulation s'est donc accompagné l'accroissement de l'autonomie des établissements français? La France met plutôt en œuvre un modèle de régulation par les processus, proposant aux établissements une série de "formes pédagogiques», supposées efficaces et pertinentes, que les échelons intermédiaires de l'administration de l'éducation les aident et les incitent fortement à adopter et à adapter à leur situation particulière. Des enseignants sont détachés à temps partiel au rectorat pour plaider dans les établissements la cause de ces «formes» et former les personnels à leur emploi. Cette pression rencontre peu de succès dans le cas du projet d'établissement ou de l'utilisation des indicateurs d'évaluation, davantage lorsque ces formes (les itinéraires de découverte, par exemple) ne paraissent pas porter en germe un contrôle accru par le niveau établissement et davantage encore lorsqu'elles n'exigent pas forcément de l'enseignant qu'il collabore avec des collègues (évaluations nationales des élèves).

Outre cette pression par la formation, ont lieu quelques rares inspections, dont l'objet est de pouvoir signaler au ministre, par l'observation d'un échantillon d'établissements, si sa politique a été mise en application. Les rapports pro- 
posent donc des observations sur cette mise en application ${ }^{6}$, mais ne s'intéressent pas aux résultats obtenus par ces politiques.

Les «formes pédagogiques» implantées en France ont de fait un double but: mieux «adapter» l'enseignement aux élèves en difficulté, faire évoluer l'enseignement vers des formes moins strictement disciplinaires.

La pertinence du premier objectif est questionnée, on l'a vu, par l'efficacité de certains "whole school designs», mais aussi par les recherches sur l'efficacité des collèges français, qui trouvent peu de traces d'interactions entre les facteurs d'efficacité et les publics d'élèves (Grisay, 1997). Les recherches sur l'hétérogénéité des classes, qui concluent que les élèves faibles gagnent moins à l'adaptation des programmes qu'ils ne perdent à être exposés de ce fait à des curriculum moins exigeants et à des condisciples plus faibles (Duru-Bellat \& Mingat, 1997) vont dans le même sens.

Celle du second ne peut être appréciée par des études empiriques à court terme. Ceci dit, quelque soit leur objectif, on voit bien que ces formes relèvent d'un mode de gouvernement où l'Etat cherche à obtenir que ses agents fassent ce qu'il souhaite plus qu'à un mode de gouvernement où il cherche à ce qu'ils atteignent les objectifs qu'il leur fixe.

Quoiqu'il en soit, cette régulation a échoué à obtenir que les enseignants d'un même établissement coordonnent leur activité. Un tiers seulement des enseignants estime qu'une coordination pédagogique existe dans leur établissement (Guillaume, 2000). Une recherche sur une échantillon de 100 collèges a montré qu'au début des années 90 en tous cas, la variation des pratiques pédagogiques déclarées par les enseignants était aussi grande au sein de chaque collège que dans l'ensemble d'un échantillon de cent collèges (Grisay, 1997). La politique d'établissement se réduit le plus souvent à «des actions ponctuelles prises en charge par de petits groupes de personnes travaillant indépendamment des autres et parfois en contradiction avec eux» (Cousin, 1998). C'est seulement, semble-t-il, là où le maintien de l'ordre pose problème, où la possibilité d'enseigner est en péril que la construction de normes communes de comportement peut apparaître comme un objectif souhaitable pour de nombreux enseignants - mais c'est loin d'être le cas pour l'activité d'enseignement elle-même (Van Zanten, 2000).

\section{Confrontation}

On peut confronter les modes de régulation français et anglo-saxons de trois façons: laquelle est le plus conforme à ce que les théories disent du fonctionnement des établissements scolaires, laquelle s'est avérée la plus efficace, laquelle est la plus cohérente?

Sur les deux premières approches, on trouvera des indications dans Meuret et Duru-Bellat (2002, 2003), et aussi dans Meuret (2002, 2003). On peut les résumer en disant que: 
- l'approche anglo-saxonne correspond clairement mieux que la française aux théories économiques des organisations (théorie du public choice, théories dîtes «principal/agent» qui fixent aux systèmes de régulation l'objectif d'atténuer l'asymétrie d'informations qui existent entre ces deux positions (Milgrom \& Roberts, 1992). L'approche française a été plutôt inspirée par des théories comme l'approche des "relations humaines» ou le "modèle organique» (Scheerens \& Bosker, 1997), qui visent à donner aux professionnels les moyens de s'adapter aux circonstances Ces théories reposent sur l'idée, certes malmenée par les résultats empiriques ${ }^{7}$, que le fonctionnement optimum d'une école varie avec son contexte.

- La satisfaction des agents, comme celle des citoyens semble ne pas avoir évolué très différemment dans les deux types de pays. En terme d'équité, la hiérarchie des trois pays varie selon les critères pris en compte et ce de façon telle qu'il semble difficile de donner l'avantage à l'un plutôt qu'à l'autre, ce qui contredit l'idée que la régulation par les résultat soit vouée à creuser les inégalités. Il y a par ailleurs, dans les évaluations nationales ou internationales, quelques indices que les performances des élèves des pays anglo-saxons se soient améliorées ces dernières années tandis que celles des élèves français seraient restées stables.

Qu'en est-il selon la troisième approche? Autrement dit, de quelles tensions, contradictions, dangers sont porteurs l'un et l'autre mode de régulation?

\section{Les tensions et contradictions du modèle anglo-saxon}

Il a fallu, on l'aura remarqué, attendre la loi No Child Left Behind pour que la régulation par les résultats soit mise en place aux USA à une échelle autre que locale, alors que l'Angleterre a mis en place d'emblée un système national. Mais c'est bien elle aujourd'hui le trait le plus distinctif des systèmes anglo-saxons.

Les critiques adressées à ce type de régulation, aussi bien en France (Chatel, 2001; Demailly, 2003) que dans les pays qui l'ont implantée (Mac Neill, 2000, par exemple) sont nombreuses: Elle conduit à enseigner de façon plus pauvre, plus ciblée sur des objectifs mesurables qui ne rendent pas justice des effets de l'action des enseignants, lesquels sont en réalité diffus, idiosyncrasiques, à long terme, bref impossibles à mesurer; Elle conduit à penser que tous les moyens sont bons pour atteindre les résultats; Elle suppose un accord politique sur la nature des «résultats» à atteindre qui est difficile à obtenir. Une sanction immédiate, comme dans les salles des marchés, pour des résultats insatisfaisants est impossible à imaginer; les enseignants ou les établissements ne peuvent être tenus pour responsables des progrès de leurs élèves puisque ce sont les élèves qui apprennent et que les enseignants se contentent de favoriser ces apprentissages; l'Etat en profite pour se défausser de ses responsabilités sur ses agents.

A ces critiques, il est possible de répondre que l'existence même d'examens suppose que l'essentiel des effets de l'enseignement soient mesurables, que la 
qualité de la régulation dépend effectivement de la qualité des examens et des tests sur lesquels elle repose, laquelle n'est pas obligée d'être médiocre; qu'une régulation par les résultats n'empêche en aucune façon de tracer des limites juridiques à l'admissible, comme c'est le cas pour les examens que passent les élèves, et même pour l'activité des entreprises, que l'idée, enfin, que la morale ne puisse s'appliquer que dans un monde sans sanctions est une idée étrange, et, à y bien réfléchir, amorale; que l'accord politique fort qui est, c'est tout à fait vrai, indispensable au fonctionnement d'un système de régulation par les résultats a pu être trouvé dans de nombreux pays; que les progrès récents des systèmes de régulation consistent effectivement à ne prendre des sanctions qu'après plusieurs années de résultats insatisfaisants et seulement si l'on n'est pas arrivé à redresser la situation par l'aide, la formation ou l'accompagnement; qu'un enseignant ne peut être rendu responsable des progrès d'un élève, mais que, la loi des grands nombres aidant, il peut l'être davantage de celui de l'ensemble de ses élèves; enfin que la régulation par les résultats ne s'assimile sûrement pas à une régulation par le marché et que, dans tous les pays où elle a été mise en place, l'Etat a revendiqué fortement la responsabilité éminente du système éducatif et d'être jugé, lui aussi, en fonction des résultats obtenus ${ }^{8}$.

Il nous semble que les tensions et les problèmes sont ailleurs: l'un, également pointé dans Demailly (2003) est le risque que le métier d'enseignant, plus dur, plus «stressant», attire moins de candidats. Les partisans de la régulation par les résultats disent que cela se traduit par une évolution vers une professionnalité plus haute et plus moderne, qui rendra au contraire le métier plus attrayant, mais cela reste à prouver. Un autre encore est que la régulation par les résultats peut entrer en conflit avec la régulation par le marché, qui tend aussi à se développer actuellement, si les critères de choix des usagers (un fonctionnement autoritaire, par exemple) jouent contre les objectifs que fixe l'Etat (l'efficacité, par exemple). A quoi il faut ajouter qu'elle peut entrer en contradiction avec elle-même: le journal Education Week s'est fait, aux Etats-Unis, l'écho de la perplexité des écoles et des parents confrontés à des jugements différents sur la qualité des écoles selon qu'ils sont portés à partir des tests des Etats ou des tests fédéraux. L'explication semble seulement que ces derniers sont plus exigeants, mais tous les acteurs demandent une mise en cohérence du système.

Il semble aussi que les Etats-Unis ne réussissent pas toujours à combiner la régulation par les résultats avec leur tradition d'expérimentation et il peut arriver que les écoles soient soumises à des injonctions au moins partiellement contradictoires. Le district étudié par Berends et al. (2002) l'illustre bien: L'administration finance pour les écoles les moins performantes (régulation par les résultats) l'implantation d'un design parmi quatre qu'elle a validés (une démarche qui, le caractère exhaustif du design mis à part, s'apparente à la régulation par les processus à la française). Le problème vient de ce que certains de ces designs prônent une pédagogie qui entre en contradiction avec les tests du district qui, selon les observateurs, incitent les écoles à se consacrer à l'apprentissage de compétences de base. 
Il faut en outre faire accepter aux acteurs l'idée qu'une autonomie de moyens s'accompagne d'une surveillance forte des résultats, idée qui paraît en général évidente aux responsables des systèmes éducatifs, mais beaucoup moins dans les établissements. La solution anglaise est, on l'a vu, que «l'autonomie de chaque établissement sera fonction de son efficacité», ce qui suppose une appétence pour la liberté, estimable sûrement mais peut être pas générale. Enfin et surtout, inciter les acteurs à être plus efficace ne les rend tels que s'ils peuvent l'être - c'est-àdire si la progression des élèves dépend effectivement de l'action, individuelle ou collective, de leurs enseignants - et s'ils savent comment l'être - c'est-à-dire s'ils connaissent et peuvent utiliser des formes d'enseignement efficaces. Il s'agît là, sans doute, de l'argument le plus sérieux contre la régulation par les résultats. Il s'appuie sur des études empiriques qui montrent que l'efficacité des établissements (mesurée en tenant sous contrôle le niveau initial de leurs élèves) n'est pas très différente d'un établissement à l'autre (sauf pour les extrémités de la distribution, les meilleurs et les moins bons) et est en grande partie commandée par les caractéristiques du public d'élèves (school mix, peer effects). La meilleure étude sur le sujet, cependant, établit que, même une fois l'effet du school mix tenu sous contrôle, il reste bien un effet établissement, petit mais significatif (Opdennaker \& Van Damme, 2001).

\section{Les tensions liées à la régulation à la française sont d'une autre nature}

Cette régulation est à certains égards contradictoire: Le ministère publie des indicateurs de performances des lycées sans que les parents puissent choisir ces derniers, et sans que rien soit entrepris envers ceux qui présentent durablement des performances faibles; les enseignants continuent d'être évalués individuellement quant on prône le travail en équipe. On multiplie les évaluations des élèves, sans évaluer les enseignants, comme si les élèves étaient seuls responsables de leurs progrès; faute d'évaluer les projets d'établissements par une mesure de leurs effets, les rectorats n'ont le choix qu'entre un contrôle de conformité, qui annule l'autonomie des établissements, et un simple contrôle de légalité, qui renonce à toute régulation.

Par ailleurs, cette politique peine à orienter vraiment les pratiques dans les établissements. La plupart des outils mis à la disposition des établissements ne sont que rarement utilisés, d'autant que leur utilité a rarement été évaluée. Ainsi, les projets d'établissement coiffent souvent de façon lâche une série disparate de projets individuels 9 . C'est d'ailleurs conforme à ce que prédit la théorie de "l'anarchie organisée» qui veut que l'on cherche plus souvent, dans les organisations, des problèmes à des solutions que des solutions à des problèmes. Dans le même temps, l'idée de l'adaptation au contexte, combinée avec une lecture normative de l'idée Crozérienne selon laquelle «l'on ne réforme pas par décret», conduit à l'idée que tout décret, toute instruction, avec lesquelles les acteurs ne sont pas d'accord n'a pas de légitimité à être appliqué: les acteurs sont les mieux placés pour savoir ce qui est bien - on n'arrête pas de le leur répéter - et toute 
contrainte ne pourrait avoir que des effets contreproductifs. Une telle idée corrompt évidemment l'idée même de gouvernement, d'administration et de service public.

Plus fondamentalement, tout se passe comme si le gouvernement du système éducatif hésitait en France entre un système centré sur les apprentissages des élèves et un système fondé sur la magistrature des enseignants et le souci de l'autorité de l'institution (Ferry, 2003), alors que les pays anglo-saxons, mais aussi scandinaves ou asiatiques, ont clairement choisi le premier.

\section{Conclusion}

Les éléments que nous avons rassemblé, et la façon dont il nous semble devoir les interpréter, ne militent pas pour l'idée que le système de régulation par les processus mis en place en France est une alternative stable à la régulation par les résultats mise en œuvre dans les pays anglo-saxons.

Cela ne signifie pas qu'elle soit à rejeter. Les responsables des systèmes éducatifs anglo-saxons eux-mêmes se sont aperçus qu'un système qui se contenterait de sanctionner les établissements ou les acteurs inefficaces et de récompenser les autres n'était pas viable. C'est une des différences entre la régulation que Mme Thatcher avait mise en place en Angleterre, fondée exclusivement sur l'idée de responsabilité, et celle qu'a mise en place le gouvernement travailliste. On a vu aussi, à travers le cas des strategies anglaises ou des designs américains, que la régulation anglo-saxonne porte parfois sur les processus. Il semble bien que la proposition d'une panoplie de formes pédagogiques, d'outils d'évaluation, voire de designs d'établissement, l'aide aux acteurs et aux établissements (sous forme d'aide, de conseil, de formation, de fourniture de ressources adaptées), doivent accompagner la régulation par les résultats.

La régulation par les processus est donc utile, et l'expérience qu'y a acquise la France peut être utile à d'autres pays, y compris anglo-saxons. Ce que montre cette comparaison, me semble-t-il, est cependant qu'alors qu'il est relativement facile d'intégrer à la régulation par les résultats une attention aux processus qui y conduisent, la considération des processus sans assez de souci des résultats conduit, elle, à un affaiblissement grave du gouvernement de l'éducation.

\section{Notes}

1 Les autres pays anglo-saxons (Australie, Canada, Nouvelle Zélande) implantent des formes de régulation voisines de celles qu'implantent l'Angleterre et les Etats-Unis, en fait plus radicalement fondées sur la compétition en Nouvelle Zélande ou dans certaines provinces australiennes. Nous décrirons l'Angleterre et non le Royaume-Uni, les règles du jeu en Ecosse ou en Irlande du Nord étant légèrement différentes.

2 Cette réforme s'est faite avec beaucoup de continuité de la part de gouvernements successifs qu'on accusait pourtant de "faire des réformes dans tous les sens». 
3 Dans cette dernière ville, c'est la Cour Suprême de l'État qui, suite à une plainte de la NAACP (National Association for the Advancement of Colored People), a obligé le district à «reconstituer» chaque année trois établissements jusqu’à ce que les inégalités de qualité entre écoles aient assez diminué (Khanna et al., 1999). Il peut par ailleurs être utile d'ajouter, à cette présentation de procédures, celle de systèmes globaux de régulation. En voici deux parmi les plus complets. Dans le district de Mineapolis, un système global incluant autonomie accrue des établissements, projet d'établissement, compte rendu, incitations est en train de s'implanter (Du et al., 1999). L'incitation consiste en ce que 10'000 à 20'000\$ sont attribués à l'établissement, en fonction de la "valeur ajoutée» aux compétences des élèves, de leur absentéisme, de leur participation à des cours de haut niveau, et de l'engagement de l'établissement dans des programmes individualisés de formation continue pour les enseignants. Le modèle multiniveau utilisé pour le calcul de la "valeur ajoutée» permet de distinguer, dans le progrès des élèves, la part de responsabilité des enseignants et celle de l'école elle même (Heistad \& Spicuzza, 2000). La performance des écoles est mesurée avec un vingtaine d'indicateurs. Les deux tiers d'entre eux sont des indicateurs d'évolution, et non d'état, pour encourager les établissements en progrès. Ils portent sur les compétences des élèves et sur le climat de l'école (respect, sécurité) (Heistadt, 2000). En Floride, un système d'accountability existe depuis 1992. Il a été durci en 1999: le "A+ plan" prévoit que tous les élèves des grades 3 à 10 passent des épreuves chaque année dans un grand nombre de disciplines, que, à certains grades, les élèves redoublent s'ils n'ont pas atteint le niveau minimum, que les écoles sont notées de $\mathrm{A}$ à $\mathrm{F}$ en fonction des résultats de ces tests, que ces notes sont publiées, que les écoles "A» sont récompensées et les écoles «F" (suite à une revendication des enseignants) aidées, sachant que, si une école obtient un $\mathrm{F}$ deux fois en 4 ans, les parents de son secteur peuvent envoyer leur enfant dans une autre école publique ou recevoir des bons d'éducation pour l'envoyer dans une école privée. Les enseignants ont obtenu que les comparaisons soient faites sur la base de la Valeur Ajoutée et non plus des performances brutes (Inman, cité par Herrington \& Kasten, 2001).

4 L'Inspecteur d'Académie est à la tête du service de l'Etat qui est responsable, dans chacun des 100 départements français, de l'enseignement dans les écoles primaires et les collèges. Ces derniers sont dirigés par un Principal, en général un ancien enseignant qui a passé un concours et reçu une formation dans ce but.

5 Il y a 27 académies en France, dont les contours sont le plus souvent ceux des régions, lesquelles comptent de deux à cinq départements. Le rectorat, qui a à sa tête un recteur, est le service déconcentré de l'Etat responsable de l'enseignement dans une académie.

6 En voici deux exemples, tirés de Bellet et Szymankiewicz (2000): «Malgré les recommandations ministérielles, qui recommandaient aux Chefs d'établissements de veiller à placer ces heures de façon pédagogiquement pertinentes [...] elles sont souvent organisées en début ou en fin de journée» (p. 33); «la constatation a pu être faîte, dans certains établissements, d'une évidente confusion entre l'aide individualisée et l'enseignement modulaire organisé pour tous les élèves d'une classe, comme entre l'enseignement modulaire et les dédoublements de classe6” (p. 36).

7 Y compris ce qui est connu sous le nom de Barclay-Calvert case, l'implantation couronnée de succès du curriculum d'une école élitiste dans celle d'un quartier populaire.

8 Cf. le célèbre «Education, education, education» de Tony Blair pendant la campagne législative de 1997 et le fait que le précédent ministre de l'éducation avait promis de démissionner si les objectifs qu'il avait fixé n'étaient pas atteints.

9 On trouvera une évaluation des Projets d'établissement dans IGEN-EVS (2001). 


\section{Bibliographie}

Bellet, A. \& Szymankiewicz, C. (2000). Suivi permanent du fonctionnement des établissements publics locaux d'enseignement, 1999-2000 Paris: MEN-IGAEN.

Berends, M., Chub, J.A., Schuyler, G., Stockly, S. \& Briggs, J.R. (2002) Challenges of Conflicting School Reforms: Effects of New American Schools in a High Poverty District. Santa Monica: Rand.

Borman, G.D., Hewes, G.M., Overman, L. \& Brown, S. (2003). Comprehensive School reform and Achievement: a Meta Analysis, Review of Educational research, 73, (2), 125-230.

Blair, J. (2000). Cincinnati Teachers to be Paid on Performance. Education Week, (September 27 th).

Chatel, E. (2001). Comment évaluer l'éducation? Lausanne: Delachaux et Niestlé.

Cousin, O. (1998). L'efficacité des collèges. Paris: PUF.

Davies, J. \& Guppy, T. (1997). Globalization and Educational Reforms in Anglo-american Democracies. Comparative Education Review, 41, (4), pp. 435-459.

Demailly, L. (2003). L'obligation de résultats en éducation, Administration et Education, (2), 67-84.

Du, Y. \& Heistad, D. (1999). School Performance Accountability in Minneapolis Public Schools. Communication au congrès de l'American Educational Research Association. Montréal.

Du Bois, P., Eaton, M., Garet, M.S. \& Miller, M. (2000). Study Design and Initial Findings for a National Evaluation of Projects Funded by the Magnet Schools Assistance Program. Communication au congrès de l'American Educational Research Association, New Orleans.

Duru-Bellat, M. \& Mingat, A. (1997). La gestion de l'hétérogénéité des publics d'élèves au collège. Dijon: IREDU (Cahiers de l'IREDU, $\left.{ }^{\circ} 59\right)$.

Duru-Bellat, M. \& Meuret, D. (2001). Nouvelles formes de régulation dans les systèmes éducatifs étrangers: autonomie et choix des établissements scolaires. Revue française de Pédagogie, (135), 173-221.

Ferry, L. (2003). Lettre à tous ceux qui aiment l'école. Paris: Odile Jacob.

Fitz-Gibbon, C. \& Stephenson, N.J. (1996). Inspecting her Majesty Inspectors: Should Social Science and Social Policy Cohere? Communication at the European Conference on Educational Research, Seville (Spain).

Grisay, A. (1997). Evolution des acquis des élèves au cours des années de collège. MEN-DEP, Dossiers Education et Formations, ${ }^{\circ}$ 87, Paris: Ministère de l'éducation.

Heistadt, D. (2000). Measuring school performance to reward effective programms in Mineapolis public schools. Communication au congrès de l'American Educational Research Association, New Orleans.

Heistad, D. \& Spicuzza, R. (2000). Measuring School Performance to Improve Student Achievement and to Reward Effective Programs. Communication au congrès de l'American Educational Research Association, New Orleans.

Herman, R. et al. (1999). An Educator's Guide to Schoolwide Reform, Arlington, VAs: Educational Research Services.

Herrington, C.D. \& Kasten, K. (2001). Florida 2001: Educational Policy Alternatives. University of North Florida: Technical report.

IGEN-VES (2001). L'autonomie de l'Etablissement Public Local d'Enseignement (EPLE) en question. Paris: MEN.

Khanna, R. et al. (1999). The History and Practice of Reconstitution in San Francisco. Communication au congrès de l'American Educational Research Association, Montréal.

Ladd, H.F. (1999). The Dallas School Accountability and Incentive Program: an Evaluation of its Impact on Students Outcomes. Economics of Education Review, 18, 1-16.

MacBeath, J. (1999). School Must Speak for Themselves. London: Routledge.

Macbeath, J., Meuret, D., Schratz, M. \& Jakobsen, L.B. (2000). Self Evaluation in European Schools: A Story of Change. London: Routledge. 
MacNeil, L. (2000). Contradictions of School Reform: Educational Cost of Standardized Testing. London: Routledge.

Meuret, D. (2003). L'équité des systèmes éducatifs, une comparaison France - Angleterre. Communication au séminaire du RAPPE, Londres.

Meuret, D. (2002). Tentative de comparaison de l'équité des systèmes éducatifs français et américains. Carrefours de l'éducation, 13, 126-151.

Meuret, D. \& Duru-Bellat, M. (2003). English and French Regulations of the Educational System: A Comparison. Comparative Education, 39, (4), 463-477.

Meuret, D. \& Morlaix, S. (2003). Conditions of Success of a Self Evaluation: Some Lesson of an European Experience. School Effectiveness and school Improvement, 14, (1), pp. 53-71.

Meuret, D. \& Duru-Bellat, M. (2002). Vers de nouveaux modes de gouvernement des systèmes éducatifs? Les leçons des comparaisons internationales. Politiques et Management publics, 20, (2), 61-81.

Meuret, D., Broccholochi, S. \& Duru-Bellat, M. (2001). Autonomie et choix des établissements scolaires: finalités, modalités, effets. Dijon: IREDU (Cahiers de l'IREDU, ${ }^{\circ} 62$ ).

Milgrom, P. \& Roberts, J. (1992). Economics, organisation and management. Prentice Hall, (trad fcse, 1997, Economie, Organisation et management. de Boeck-Presses Universitaires de Grenoble)

Murphy, J. \& Beck, L.G. (1995). School Based Management as School Reform. Thousand Oaks, CA: Corwin Press, A Sage publication.

Nelson, B., Berman, P. et al. (2000). The State of Charter Schools. Fourth Year Report. Washington DC. OERI, US Department of education.

OFSTED [Office for Standard in Education] (2000). Inspecting Schools, The framework. London: OFSTED.

Opdenakker, M.C. \& Van Damme, J. (2001). Relationship between School Composition and Charactéristics of School Process and their Effect on Mathematic Achievement. British Educational Research Journal, 27, (4), 407-432.

Pedrosko, J.M. \& Lindle, J.C. (1999). State Mandated School Reform in Kentucky: Year Three of a Longitudinal Study. Communication au congrès de l'American Educational Research Association, Montréal.

Riley, K. \& Rowles, D. (1997). Inspection and School Improvement in England and Wales. In T. Townsend (Ed.), Restructuring and Quality: Issues for Tomorrow's Schools. London: Routledge.

Rosenthal, L. (2004). Do School Inspections Improve School Quality? OFSTED inspections and school examinations results in the UK. Economics of Education Review, 23, 143-151.

Saunders, L. (1999). Who or what is School «Self»-Evaluation for? School Effectiveness and School Improvement, 10, (4), 414-429.

Schriewer, J. (1997). L'éducation comparée, mise en perspective historique d'un champ de recherche. Revue française de Pédagogie, (121), 9-28.

Skrla, L., Scheurich, J., Johnson, J.F. \& Kosschorek, J.W. (2000). Accountability for Equity: Can State Policy Leverage Social Justice? Communication au congrès de l'American Educational Research Association, New Orleans.

Slavin, R.E., Madden, N.A. et al. (1996). Success for all: A summary of research. Journal of Education for Students placed at Risk, 1, (1), 41-76.

Springfield, S. (2000). A Synthesis and Critique of Four Recent Reviews of Whole School Reform in the United States. School Effectiveness and School Improvement, 11, (2), 259-269.

Stoll, L. \& Myers, K. (Eds.) (1997). No Quick Fixes. London: Falmer.

Thomas, S. (2001). Innovations in School Evaluation: An English Case Study of the Lancashire Value Added Project. Communication au Séminaire du réseau RAPPE sur la régulation des systèmes éducatifs.

Van Zanten, A. (2001). L'école de la périphérie. Paris: PUF. 
Wilson, T. A. (2001). Legitimizing Practitioner Knowledge: Key to Effective Accountability and School Improvement. Communication au XIVème congrès School Effectiveness and School Improvement, Toronto.

Wragg, E.C. et al. (1999). The Role of the Headteacher in Addressing the Problem of Incompetent Teachers. Communication au congrès de l'American Educational Research Association, Montréal.

Mots clefs: Politique de la formation,, pilotage des systèmes de formation, administration de la formation, autonomie de l'établissement scolaire, surveillance de l'établissement scolaire, comparaison entre pays

\section{Die Steuerung des Bildungssystems in Frankreich und in den angelsächsichen Ländern: ein Vergleich}

\section{Zusammenfassung}

In den meisten Bildungssystemen hat die Autonomie der Einzelschulen in den letzten 20 Jahren zugenommen. Infolgedessen mussten diese Staaten eine Steuerungspolitik etablieren, um die Aktivitäten der Akteure in die gewünschte Richtung zu lenken. Während England und die USA eine an den Resultaten (outcomes) orientierte Steuerung einführten (positiv oder negativ anreizorientierte Mittelvergabe in Abhängigkeit von den Leistungen der Schulen oder der Lehrpersonen) setzt Frankreich eher auf eine Steuerung der Prozesse (Anreize zur Durchsetzung von pädagogischen Methoden und Verfahrensweisen, die auf nationaler Ebene als angemessen und «nachhaltig» beurteilt werden).

Diese Politiken werden im vorliegenden Beitrag beschrieben und unter der Perspektive der Kohärenz und der Entwicklungsrichtung, die sie auslösen, miteinander verglichen. Zu prüfen ist, ob das französische Modell ein praktizierbares Modell, eine Alternative zum angelsächsischen Modell sei. Die Antwort ist vorwiegend negativ; gleichzeitig wird deutlich, dass die angelsächsischen Länder die beiden Steuerungsmodelle zunehmend vermischen.

Schlagworte: Bildungspolitik, Steuerung des Bildungssystems, Bildungsverwaltung, Schulautonomie, Schulaufsicht, Ländervergleich

\section{II pilotaggio del sistema formativo in Inghilterra e nei paesi anglosassoni: un confronto}

\section{Riassunto}

Nella maggior parte dei sistemi scolastici l'autonomia delle singole scuole è aumentata negli ultimi anni. Ci si è pertanto dovuti dotare di una nuova politica capace di indirizzare le attività degli attori del sistema nella direzione voluta. 
Mentre l'Inghilterra e gli Stati Uniti hanno adottato una politica basata sui risultati (outcomes - distribuzione di incentivi positivi e negativi in funzione dei risultati degli istituti scolastici), la Francia tende piuttosto ad un pilotaggio dei processi (la sollecitazione a mettere in atto dei dispositivi pedagogici considerati pertinenti a livello centrale). Queste politiche vengono discusse nell'intento di chiarire se il modello francese sia un'alternativa percorribile rispetto a quello anglosassone. La risposta è piuttosto negativa, considerato anche il fatto che gli stessi paesi anglosassoni tendono a mescolare le due modalità di pilotaggio.

Parole chiave: Politica formativa, pilotaggio di sistemi formativi, amministrazione scolastica, autonomia scolastica, sorveglianza scolastica, confronto tra stati

\section{A Comparison of Regulation Policies in Education Systems in France and in Anglo-Saxon Countries.}

\section{Summary}

The autonomy of the schools increased in most educational systems for the last twenty years. So, educational systems had to implement regulation policies in order to orient the action of the agents towards the nation's aims. While England and the United States implemented a regulation by results (incentives linked to school's or teacher's results), France relies on a regulation by process (incentives to implement 'pedagogical forms' which are supposed to be relevant). These two policies are described, then they are confronted with regard to their internal coherence and tensions, with in mind the question of the French model being a viable one, able to offer an option to the anglo-saxon model. The answer tends to be negative, even if it becomes clear that anglo-saxon countries import some elements of regulation by process in their own functioning.

Key words: educational policy, orientation of educational systems, school administration, school autonomy, school supervision, comparison of countries 